\title{
The impact of cold chain temperature abuses on the quality of frozen strawberries (Fragaria $\times$ ananassa)
}

\author{
Rui M. S. Cruz ${ }^{\mathrm{a}}$, Margarida C. Vieira ${ }^{\mathrm{a}}$, and Cristina L. M. Silva ${ }^{\mathrm{b}}$ \\ ${ }^{a}$ CIQA-Centro de Investigação em Química do Algarve and Instituto Superior de Engenharia, Universidade do \\ Algarve, Campus da Penha, 8005-139 Faro, Portugal. E-mail: mvieira@ualg.pt and rcruz@ualg.pt \\ b CBQF-Centro de Biotecnologia e Química Fina, Escola Superior de Biotecnologia, Centro Regional do Porto \\ da Universidade Católica Portuguesa, Rua Dr. António Bernardino de Almeida, 4200-072 Porto, Portugal. \\ ${ }^{*}$ Corresponding author \\ clsilva@porto.ucp.pt \\ TEL: $+351-22-5580058$ \\ FAX: $+351-22-5090351$
}

Received: 25 May 2012; Published online: 18 April 2013

\begin{abstract}
The quality of frozen foods can be negatively affected if improper storage and distribution temperatures are allowed. The objective of this study was to investigate the effect of freeze-thaw cycles, which may occur in the cold chain, on colour (Lab, Total Colour Differences (TCD), chroma and hue angle) and vitamin $\mathrm{C}$ (ascorbic and dehydroascorbic acids) content of frozen strawberries (Fragaria $\times$ ananassa, Duschesne, cv. Selva). A plan of temperature abuses (TAs) was established, based on a real situation, and applied to frozen strawberries during a four month frozen storage period. The results showed that the lightness $(L)$ was the only parameter that was not significantly affected by range of TAs studied. The colour showed some variation on the parameters a, b, TCD, chroma and hue angle. During TAs, ascorbic acid decreased about $75 \%$ and dehydroascorbic acid increased $73 \%$. The non-abused strawberry samples showed better overall appearance than the abused samples. This work contributes to an understanding of the quality changes of frozen strawberries that might occur during frozen storage and cold chain distribution.
\end{abstract}

Keywords: strawberries, frozen storage, temperature abuses, vitamin C, colour. 
$60 \mid$ Cruz et al.

\section{Nomenclature}

a Colour space co-ordinate, represents red to green

$b \quad$ Colour space co-ordinate, represents blue to yellow

L Colour space co-ordinate, represents black to white

AA Ascorbic acid

DFQ Fluorophore 3-(1,2-dihydroxyethyl)furo(3,4-b)quinoxaline-1-one

DHAA Dehydroascorbic acid

DKGA 2,3-diketogulonic acid

IAA Isoascorbic acid

OPDA 1,2-phenylenediamine dihydrochloride

TAs Temperature abuses

TCD Total colour difference parameter

\section{Introduction}

Strawberries (Fragaria $\times$ ananassa) are normally consumed fresh and in processed products such as jams, juices and frozen products. The large scale of frozen strawberry production can be attributed to the short shelf-life of fresh fruit and to its very varied use as a frozen product (Oszmiański, Wojdylo, \& Matuszewski, 2007; Wicklund et al., 2005). Freezing strawberries is known as a good method for increasing shelf-life, but berries undergo quality changes throughout the whole frozen food chain, i.e. freezing, subsequent frozen storage and thawing (Kmiecik, Jaworska, \& Lisiewska, 2000). Freezing is one of the most important methods for the quality preservation of fruits and vegetables during long-term storage (Cano, 1996). The main factors affecting the quality of frozen fruits and vegetables are storage temperature, storage time and temperature fluctuations during distribution and storage. These factors may affect texture, flavor, appearance, colour, and nutritional properties (Blond \& Le Meste, 2004). Quality preservation of frozen products is practically maintained with cold storage temperatures ranging from -25 to $-40{ }^{\circ} \mathrm{C}$ (Cano, 1996). The vitamin $\mathrm{C}$ content, besides being an indicator of nutrient value, is, in the case of frozen fruits and vegetables, a reliable index for estimating the quality deterioration at any point of the marketing route of a product to its final destination, the consumer (Fennema, 1977; Giannakourou \& Taoukis, 2003). Colour is another quality attribute that strongly influences the consumer in the final purchase of a product (Hunter Lab, 2000). Fluctuating temperatures during the distribution of frozen products and the cyclic nature of refrigeration systems stimulate crystal growth (Blond \& Le Meste, 2004). From the factory to the consumer, frozen products are submitted to different types of scenarios along all the steps of the distribution chain. In the factory, distribution centres, transportation trucks, loading/unloading, the frozen product can be affected if the storage temperatures are not appropriate. Thus, maintaining a suitable storage temperature is a key factor in order to maintain food quality. Studying the behavior of frozen products submitted to undesirable conditions along the cold chain can be an important outcome, since it will allow testing the product stability under those conditions (Cruz, 2009). Therefore, simulating the effect of temperature changes on frozen food products along the cold chain will help to provide better quality frozen food products to the final consumer. The objective of this work was to investigate the effects of TAs on the colour and vitamin $\mathrm{C}$ content of frozen strawberries. The findings will contribute to an understanding of the changes that might occur during frozen storage and distribution in the food chain, and to the consideration of further regulatory measures. 


\section{Materials and Methods}

\section{$2.1 \quad$ Frozen samples}

Fresh strawberries (Fragaria ×ananassa, Duschesne, cv. Selva) were obtained from a cultivated area in Covilhã, Portugal. Strawberries were then selected, washed and cut in small cubes $\left(1 \mathrm{~cm}^{3}\right)$. The samples were then frozen in an air blast freezer (Armfield FT 36, Hampshire, England) at $-35{ }^{\circ} \mathrm{C}$ for $10 \mathrm{~min}$, and $250 \mathrm{~g}$ of frozen strawberry cubes were packed in low density polyethylene bags $(20 \mathrm{~cm} \times 18 \mathrm{~cm})$ and stored at $-21{ }^{\circ} \mathrm{C}$ (Haier HF-248, Wettenberg, Germany).

\subsection{Plan of temperature abuses}

A plan of TAs was established, based on a real situation for a four month period (Oliveira, Cruz, Vieira, Silva, \& Gaspar, 2009), and applied to the frozen strawberries (Figure 1). The samples were stored in vertical freezers (Haier HF248, Wettenberg, Germany) and analysed in each step of the plan (S1-S6 corresponds to production and distribution; S7-S20 corresponds to consumer product purchase and storage). The temperature thermal centre of the samples was assessed with a type $\mathrm{T}$ thermocouple and recorded with a data acquisition system (Delta-T Devices DL2 e, Cambridge, England).

\subsection{Colour measurement}

Colour was evaluated in the Hunter system in terms of Lab values, hue angle (tone or tint equation (1)) and chroma (saturation - equation (2)) with a tristimulus colorimeter (Dr Lange Spectro-colour, Berlin, Germany) (Hunter Lab, 2000). The colorimeter ( $\mathrm{d} / 8^{\circ}$ geometry, illuminant D65, $10^{\circ}$ observer) was calibrated against a standard ceramic white tile $(\mathrm{X}=84.60, \mathrm{Y}=89.46$, $\mathrm{Z}=93.85$ ) and a standard ceramic black tile $(\mathrm{X}=4.12, \mathrm{Y}=4.38, \mathrm{Z}=4.71)$. The colour changes were interpreted by determining the TCD (Total Colour Differences) (equation (3)) where $\Delta L, \Delta a$ and $\Delta b$ are calculated by equations (4). Measurements were taken in triplicate.

$$
\begin{gathered}
\text { Hue angle }=\left(\tan ^{-1} b / a\right) \\
\begin{aligned}
\text { Chroma } & =\left(a^{2}+b^{2}\right)^{1 / 2} \\
\text { TCD }=\sqrt{(\Delta L)^{2}+(\Delta a)^{2}+(\Delta b)^{2}} & \\
\Delta L & =L-L_{0} \\
\Delta a & =a-a_{0} \\
\Delta b & =b-b_{0}
\end{aligned}
\end{gathered}
$$

where $L_{0}, a_{0}$ and $b_{0}$ are the measurements of the frozen samples before TAs, and L, a and b the individual readings at each step of the TAs plan.

\section{$2.4 \quad$ Ascorbic acid and dehydroascorbic acid analysis}

Based on a method previously reported by Zapata and Dufour (1992), ascorbic acid (AA) (Riedel-de Haën, Berlin, Germany) content was determined by reverse phase ion interaction high performance liquid chromatography HPLC UV detection, using isoascorbic acid (IAA) (Fluka, Berlin, Germany) as internal standard. Dehydroascorbic acid (DHAA) (Sigma, Berlin, Germany) was as well detected as fluorophore 3-(1,2-dihydroxyethyl)furo[3,4b]quinoxaline-1-one (DFQ), after pre-column derivatization with 1,2-phenylenediamine dihydrochloride (OPDA) (Sigma, Berlin, Germany). The mobile phase was composed by $13.61 \mathrm{~g}$ of potassium dihydrogen phosphate (Merck, Darmstadt, Germany), $3.64 \mathrm{~g}$ of cetrimide (Fluka, Berlin, Germany) and $2 \mathrm{~L}$ of methanol-ultrapure water $(5: 95, \mathrm{v} / \mathrm{v})$. The eluent was then filtered in a $0.45 \mu \mathrm{m}$ membrane (Macherey-Nagel, Porafil) and degassed in an ultrasonic bath for 15 min. The HPLC apparatus comprised a controller (LKB-2152, Bromma, Sweden), a solvent pump (LKB-2150, Bromma, Sweden), an injection valve with a $20 \mu \mathrm{L}$ sample loop, a guard pre-column (Macherey-Nagel, Chromcart Nucleosil 100-10 C18) followed by a reverse phase column (Macherey-Nagel, Chromcart 100-10 Nucleosil, $250 \times 4.6 \mathrm{~mm}$ ), and a UV detector (LKB2153, Bromma, Sweden). Each sample was homogenized with an Ultra-turrax (IKA T25 Janke \& Kunkel, Staufen, Germany) in $20 \mathrm{~mL}$ of methanol-ultra pure water $(5: 95, \mathrm{v} / \mathrm{v})$ for 5 
$62 \mid$ Cruz et al.

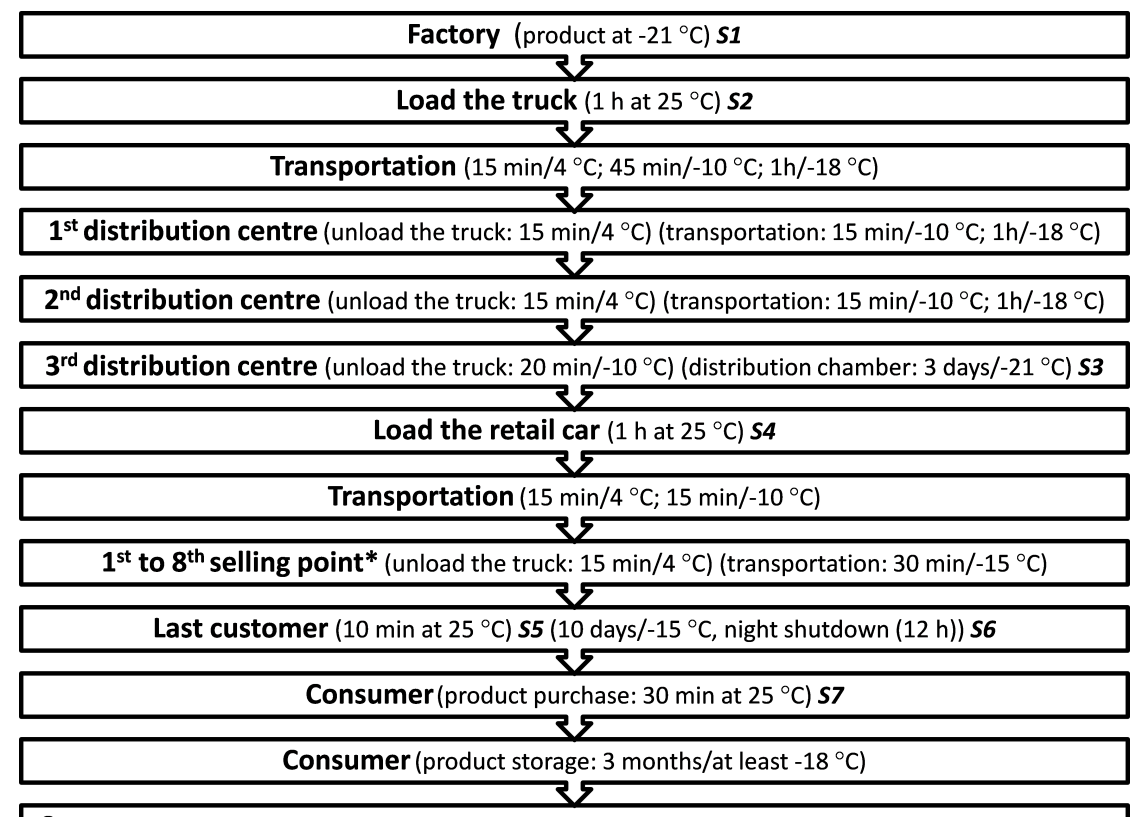

Consumer (plus 5 door openings of 3 min and 1 door opening of 30 min per week (shopping simulation)) S8-S2O

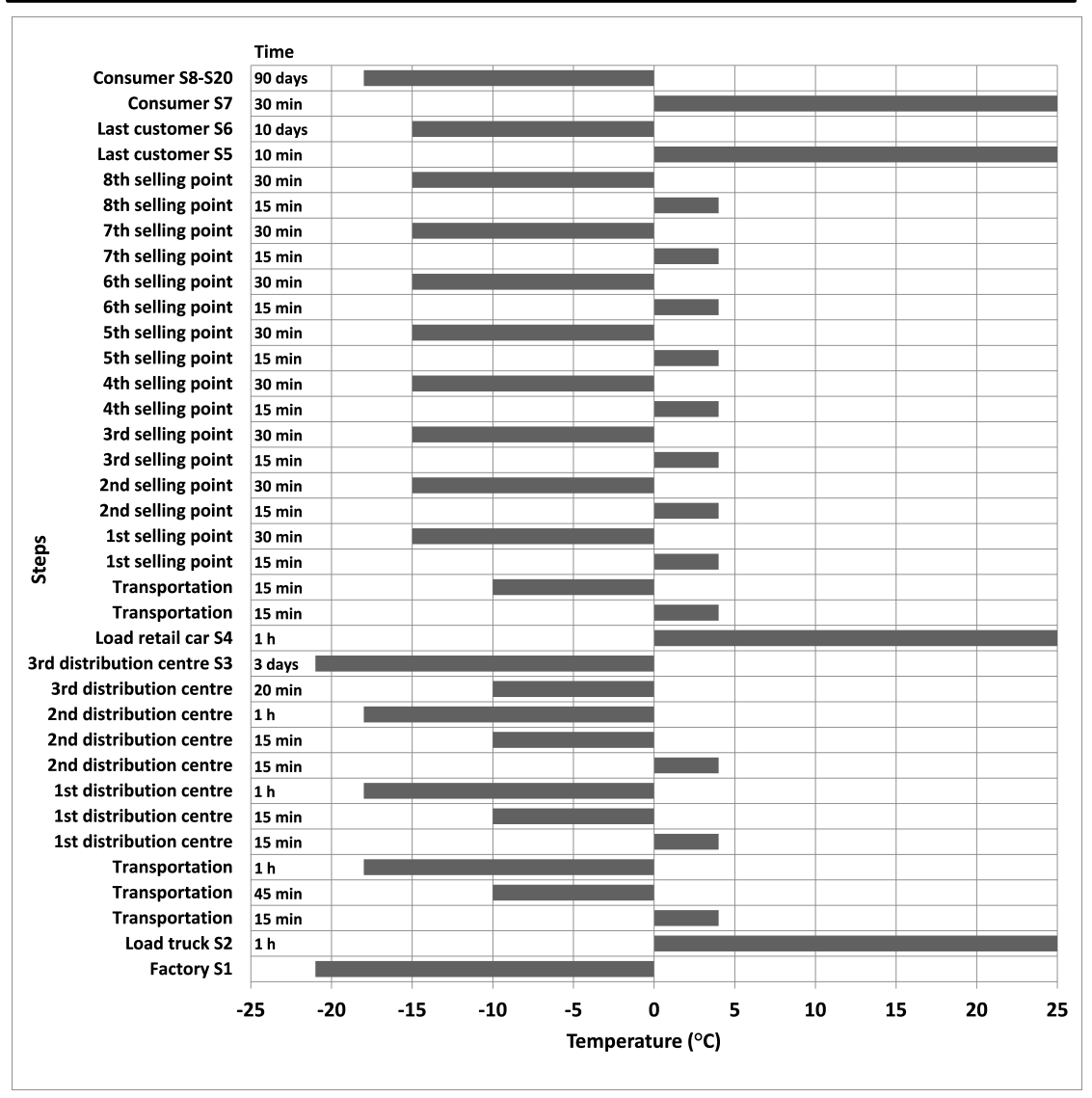

Figure 1: Plan of temperature abuses. S1 to S20- steps of analysis; ${ }^{*}$ These two steps were repeated 8 times. 
$\min$ at $8000 \mathrm{rpm}$. Afterwards, $5 \mathrm{~mL}$ were transferred to a $20 \mathrm{~mL}$ volumetric flask and $1 \mathrm{~mL}$ of IAA standard solution $(0.03 \mathrm{~g} / 50 \mathrm{~mL})$ was added. The $\mathrm{pH}$ was adjusted with $\mathrm{HCl}$ (Merck) to obtain final values between 2.20 and 2.45 . The volume was made up to $20 \mathrm{~mL}$ with methanolultra pure water $(5: 95, \mathrm{v} / \mathrm{v})$. The content was centrifuged (Sigma 3 K20, Osterode, Germany) for $5 \mathrm{~min}$ at $8.720 \times \mathrm{g}$ and $4{ }^{\circ} \mathrm{C}$. Subsequently, $3 \mathrm{~mL}$ were transferred to another tube with 1 $\mathrm{mL}$ of OPDA (Sigma) $(0.03 \mathrm{~g} / 50 \mathrm{~mL})$ which was daily prepared and maintained in the dark. The mixture was then stirred in a vortex and placed in the dark at room temperature for 40 min. Then the mixture was filtered, using a $0.45 \mu \mathrm{m}$ membrane (Millipore), the first millilitre was discarded and $20 \mu \mathrm{L}$ were injected in the HPLC. The wavelength detector was set to $348 \mathrm{~nm}$ and after elution of DHAA, the wavelength was shifted to $262 \mathrm{~nm}$ for AA and IAA detection. The vitamin C total content was assumed to be the sum of the two biological active forms (ascorbic and dehydroascorbic acids). The experiments were run in triplicate.

\subsection{Statistical analysis}

A one-way analysis of variance (ANOVA) was performed to determine significant differences between each step of the planned TAs. The Least Significant Difference (LSD) test was run to determine the significant differences between the control (without abuses) and each treatment. Evaluations were based on a significance level of $5 \%$ and calculations were performed using the statistical software SPSS 17.0.

\section{Results and Discussion}

The experimental values for frozen strawberry colour and vitamin $\mathrm{C}$ content, before undergoing the planned TAs are shown in Table 1. From Figure 2, it can be observed that the $L a b$ colour parameters were practically maintained along the TAs plan. The $L$ parameter (fruit darkening indicator) showed no significant differences $(P>$ 0.05 ) between the control and all the steps until the end of the plan (approximately $L=25$ ), meaning that the lightness was not affected by
Table 1: Frozen strawberries' colour and vitamin $\mathrm{C}$ content before the plan of temperature abuses.

\begin{tabular}{|c|c|}
\hline$L$ & $25.82 \pm 0.19$ \\
\hline$a$ & $8.57 \pm 1.07$ \\
\hline$b$ & $6.94 \pm 0.32$ \\
\hline Chroma & $11.04 \pm 1.03$ \\
\hline Hue $\left({ }^{\circ}\right)$ & $39.14 \pm 2.17$ \\
\hline $\begin{array}{l}\text { AA } \\
\left.\text { (mg.100 } \mathrm{g} \mathrm{fw}^{-1}\right) \\
\text { DHAA }\end{array}$ & $42.36 \pm 13.73$ \\
\hline $\begin{array}{l}(\mathrm{mg} .100 \mathrm{~g} \mathrm{fw} \\
\text { Total vitamin } \mathrm{C}\end{array}$ & $42.56 \pm 13.97$ \\
\hline$\left(\mathrm{mg} .100 \mathrm{~g} \mathrm{fw}^{-1}\right)$ & $84.93 \pm 5.60$ \\
\hline
\end{tabular}

the imposed temperature fluctuations (Figure 2). Throughout the plan of TAs the results showed some variations $(P<0.05)$ in the $a$ and $b$ parameters between the samples with and without TAs (Figure 2). The samples redness ( $a$-value) in the $S 3$ (approximately $a=15)$ and $S 4(a=13)$ steps (simulation at the chamber of the third distribution centre and loading the retail car) showed the highest differences compared with the nonabused samples $(a=11)$. The frozen strawberry yellowness (b-value) showed the highest differences between the control $(b=7)$ and the first, at sixth and ninth week at the consumer $(b=4)$. The frozen strawberry TCD values showed some fluctuations along the plan with an average value of 4 (Figure 3). Gormley, Walshe, Hussey, and Butler (2002) also reported TCD values in the same range in frozen strawberries submitted to temperature fluctuations. The chroma showed significant differences $(P<0.05)$ between the control and steps $S_{3}$ and $S_{4}$ (Figure 3), corroborating the results observed for the $a$-value, meaning that on those steps the samples were redder. Although the hue angle (Figure 4) showed significant differences $(P<0.05)$ between the control $\left(\mathrm{H}=30^{\circ}\right)$ and the $S 8$ and $S 13$ steps (first and sixth week at the consumer), along the plan of TAs, the values were maintained between $25^{\circ}$ $35^{\circ}$. The frozen strawberries practically maintained its colour. This is probably related to the low moisture loss during the plan of TAs that, if higher, would promote oxidative browning reac- 
$64 \mid$ Cruz et al.

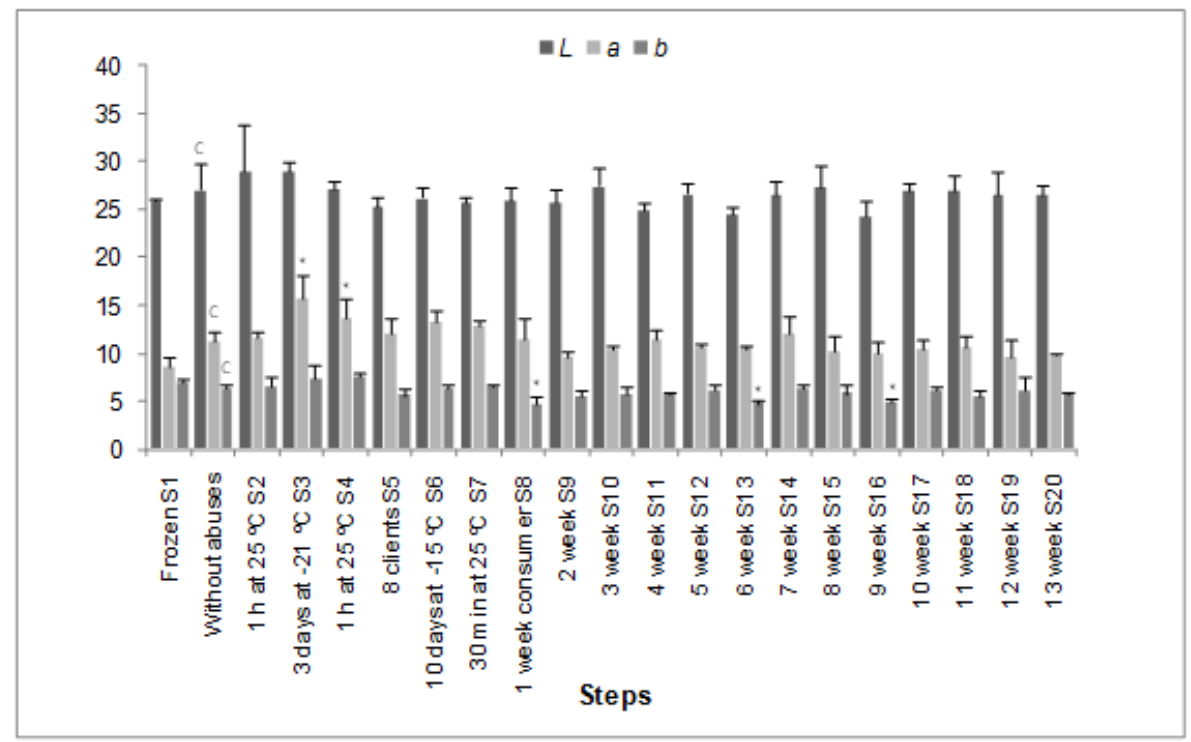

Figure 2: Frozen strawberries' Hunter Lab parameters along the temperature abuses of storage. Bars represent mean \pm standard deviation from three replicates. C-control; * significant differences with control $(\alpha=0.05)$

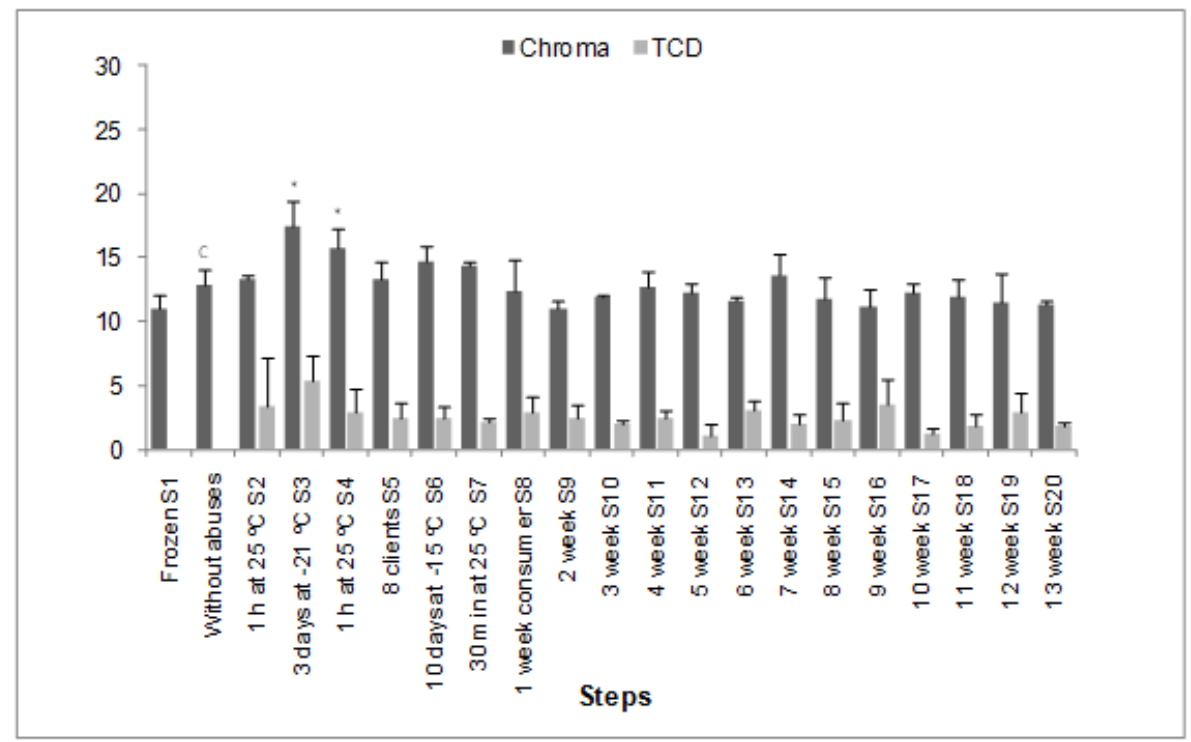

Figure 3: Frozen strawberries' TCD and chroma parameters along the temperature abuses of storage. Bars represent mean \pm standard deviation from three replicates. C-control; *significant differences with control $(\alpha=0.05)$. 


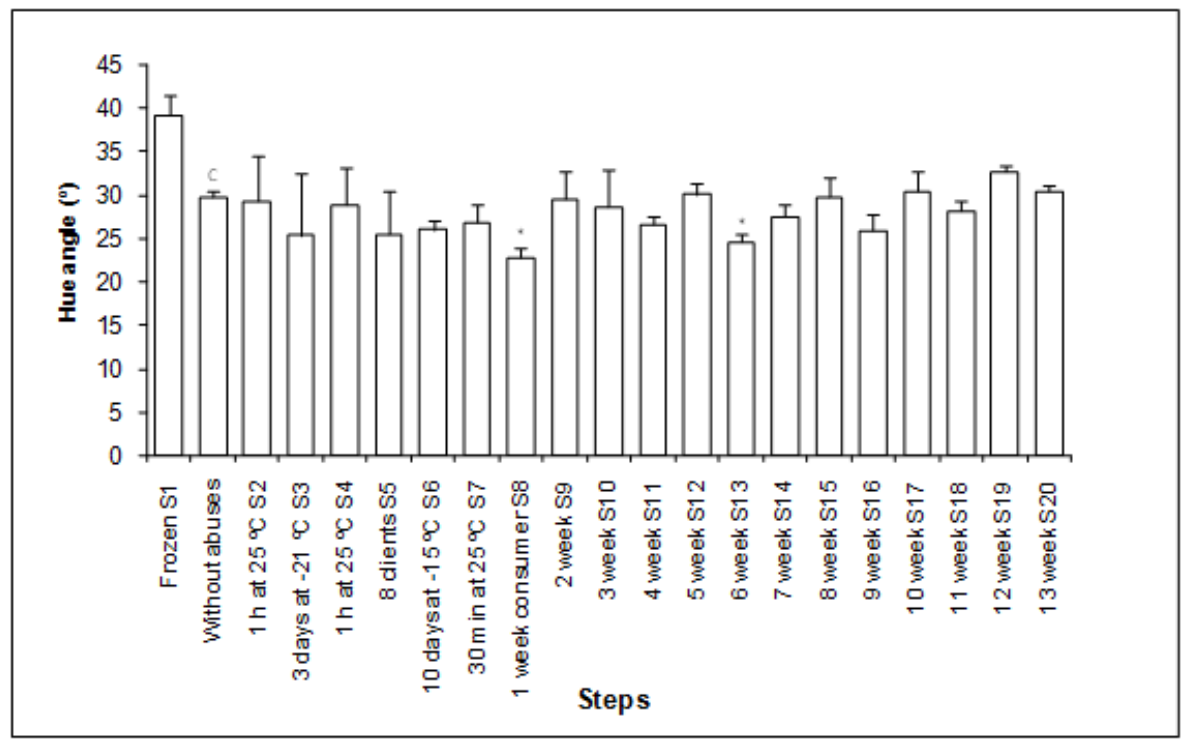

Figure 4: Frozen strawberries' hue angle along the temperature abuses of storage. Bars represent mean \pm standard deviation from three replicates. C-control; * significant differences with control $(\alpha=0.05)$.

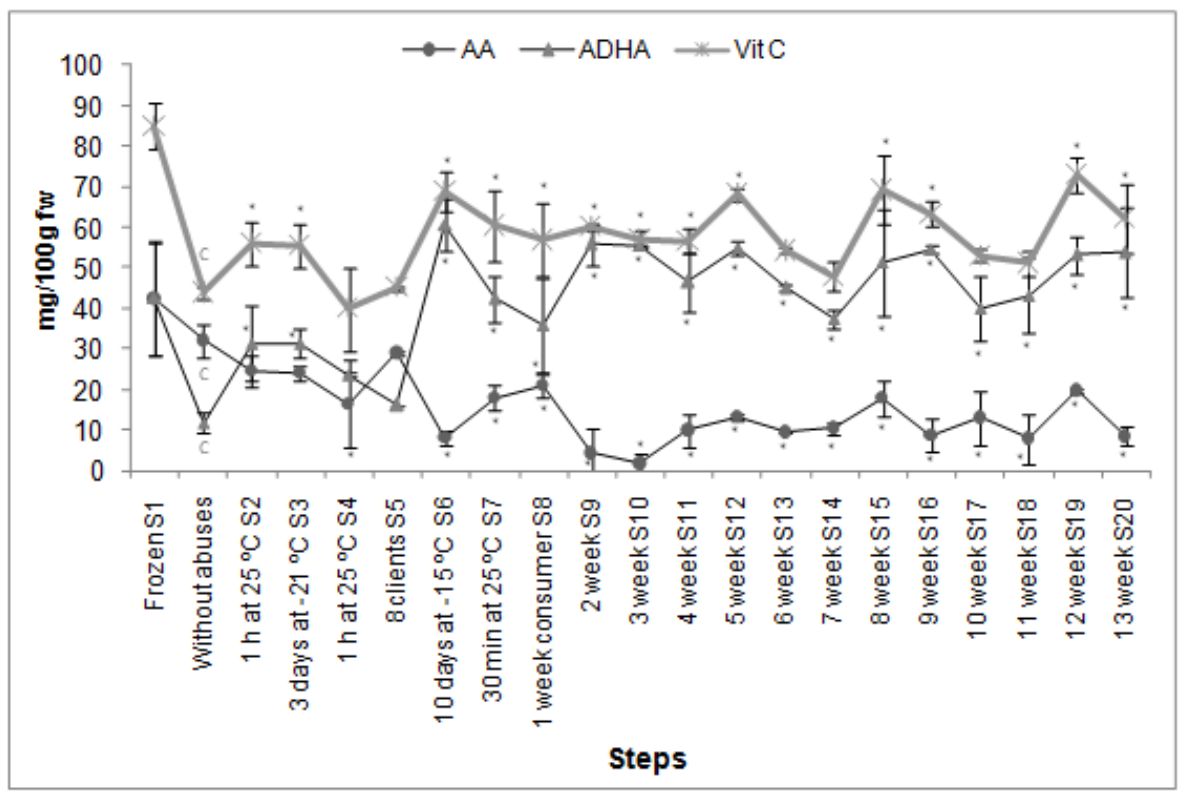

Figure 5: Frozen strawberries' ascorbic acid, dehydroascorbic acid and total vitamin C evolution along the temperature abuses of storage. Bars represent mean \pm standard deviation from three replicates. Ccontrol; *significant differences with control $(\alpha=0.05)$. 
$66 \mid$ Cruz et al.

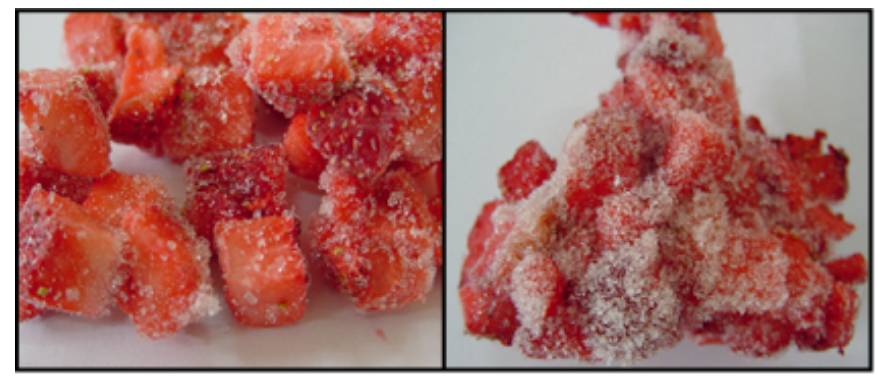

Figure 6: Frozen strawberries at the end of the plan of temperature abuses: a) non-abused sample; b) abused sample.

tions and, consequently, contribute to darker and less red strawberries (Nunes, Brecht, Morais, \& Sargent, 2005). In terms of vitamin $\mathrm{C}$, the results showed some fluctuations $(P<0.05)$ in both active forms (Figure 5). In terms of AA, $75 \%$ losses were observed along the plan of TAs, although its content was constant during consumer storage. This result was probably due to the temperature increase and the consequent conversion of $\mathrm{AA}$ to DHAA. The DHAA content increased around $73 \%$ along the TAs, so by the end of the storage period this was the prevalent form of this vitamin. This indicates that the known reversible equilibrium is being replaced by an irreversible consecutive reaction. Thus, the vitamin $\mathrm{C}$ total value was not impaired by the imposed TAs since DHAA still has vitamin $\mathrm{C}$ biological activity. The diketogulonic acid (DKGA) is probably the next product to be formed as the DHAA lactone ring is very susceptible of hydrolysis. The AA losses $(75 \%)$ occurred mainly during the first fifteen days of storage when the TAs were the highest (transportation from factory to store; S1-S6). Sahari, Boostani, \& Hamidi (2004) also reported losses of ascorbic acid in frozen strawberries during the first 15 days of storage $\left(31.4 \%\right.$ at $\left.-12{ }^{\circ} \mathrm{C}\right)$. These results are also in agreement with those of Ibanez, Foin, Cornillon, and Reid (1996) who reported a major decrease in the ascorbic acid content of frozen strawberries in the first 15 days of storage. This behavior is probably due to the increase in concentration of solutes that occurs in the unfrozen phase during freezing and temperature fluctuations (Thompson \& Fennema, 1971). The total vitamin $\mathrm{C}$ content of frozen straw- berries showed some variation throughout the planned temperature abuses, although its value from the beginning until the end of the plan was practically maintained, even if converted from one form to the other. The variation is mainly due to the temperature fluctuations and can also be associated with the initial variability of vitamin $\mathrm{C}$ in strawberries. The results in this work are in agreement with those reported by Gormley et al. (2002), in which the vitamin C content of frozen strawberries, subjected to temperature fluctuations, was practically maintained when compared to the control. Sousa, Canet, Alvarez, and Tortosa (2005) also showed, in a study with frozen raspberries, that temperature is a much more important factor than storage time in determining quality loss. Short term frozen storage with fluctuations caused a decrease in the ascorbic acid content of the raspberry fruits. The retention of ascorbic acid in frozen products is thus strongly dependent on their temperature history. In a study simulating the same plan of TAs, frozen watercress slabs showed similar results (Cruz, Vieira, \& Silva, 2009). The vitamin $\mathrm{C}$ content and the colour parameters showed some fluctuations along the plan of temperature abuses. Moreover, the non-abused samples showed a much better appearance than the abused samples, in which the strawberry cubes were covered with a mass of ice (Figure 6) due to drip loss and water refreezing. 


\section{Conclusions}

In conclusion, frozen strawberries show, except in particular steps of the planned TAs, some storage stability in terms of colour and vitamin C. This work contributes to an understanding of the quality changes of frozen strawberries that might occur during frozen storage and cold chain distribution. Testing frozen products under these scenarios, with different storage and distribution time-temperature conditions, is an important outcome since it will help with further optimisation of cold chain quality management programs and consequently improve the final quality of frozen products.

\section{References}

Blond, G., \& Le Meste, M. (2004). Principles of frozen storage. In Y.E. Hui, P. Cornillon, I.G. Legaretta, M.H. Lim, K.D. Murrell \& W. Nip (Eds.). Handbook of frozen foods (pp. 40-48). New York: Marcel Dekker.

Cano, M. P. (1996). Vegetables. In L.E. Jeremian (Ed.). Freezing effects on food quality.(pp.247-298) New York: Marcel Dekker.

Cruz, R. M. S. (2009). Development of innovative pre-treatments for watercress (Nasturtium officinale) freezing process. $\mathrm{PhD}$ thesis. College of Biotechnology-Portuguese Catholic University, Porto.

Cruz, R. M. S., Vieira, M. C., \& Silva, C. L. M. (2009). Effect of cold chain temperature abuses on the quality of frozen watercress (Nasturtium offcinale R. Br.) Journal Of Food Engineering, 94 (1), 90-97. doi:10. 1016/j.jfoodeng.2009.03.006

Fennema, O. (1977). Loss of Vitamins in Fresh and Frozen Foods. Food Technology, $31(12), 32-\&$.

Giannakourou, M., \& Taoukis, P. (2003). Kinetic modelling of vitamin $\mathrm{C}$ loss in frozen green vegetables under variable storage conditions. Food Chemistry, 83(1), 33-41. doi:10.1016/S0308-8146(03)00033-5

Gormley, R, Walshe, T, Hussey, K, \& Butler, F. (2002). The effect of fluctuating vs. constant frozen storage temperature regimes on some quality paramets of selected food products. Lebensmittel-Wissenschaft UndTechnologie-Food Science and Technology, 35(2), 190-200. doi:10.1006/fstl.2001.0837

Hunter Lab. (2000). Applications notes. 12 (5), 1-8.

Ibanez, E., Foin, A., Cornillon, D., \& Reid, D. S. (1996). Kinetics of colour change and ascorbic acid loss in selected frozen fruits and vegetable. Presented at the Institute of Food Technologists Annual Meeting, New Orleans, USA.

Kmiecik, W., Jaworska, G., \& Lisiewska, Z. (2000). Effect of sucrose, L-ascorbic acid and pectin on the quality of frozen strawberries. Electronic Journal of Polish Agricultural Universities, 3( 2).

Oliveira, S. R., Cruz, R. M. S., Vieira, M. C., Silva, C. L. M., \& Gaspar, M. N. (2009). Enterococcus faecalis and Pseudomonas aeruginosa behaviour in frozen watercress (Nasturtium officinale) submitted to temperature abuses. International Journal Of Refrigeration-Revue Internationale du Froid, 32(3), 472-477. doi:10 . 1016/j.ijrefrig.2008.08.002

Oszmiański, J., Wojdylo, A., \& Matuszewski, P. (2007). Polyphenols compounds changes in the industrial production process of concentrated strawberry juice. Food. Science. Technology. Quality., 50, 94-104.

Sousa, M., Canet, W, Alvarez, M., \& Tortosa, M. (2005). The effect of the pre-treatments and the long and short-term frozen storage on the quality of raspberry (cv. Heritage). European Food Research and Technology, 221(1-2), 132-144. doi:10 . 1007 / s00217 005-1189- 1

Thompson, L. U., \& Fennema, O. (1971). Effect of freezing on oxidation of l-ascorbic acid. Journal of Agricultural and Food Chemistry, 19(1), 121-124. PMID: 5540746. doi:10.1021/jf60173a018. eprint: http:// pubs.acs.org/doi/pdf/10.1021/jf60173a018

Wicklund, T, Rosenfeld, H., Martinsen, B., Sundfor, M., Lea, P, Bruun, T, ... Haffner, K. (2005). Antioxidant capacity and colour of strawberry jam as influenced by cultivar and storage conditions. LWT-Food Science 
$68 \mid$ Cruz et al.

And Technology, 38(4), 387-391. doi:10 . 1016/j.lwt.2004.06.017

Zapata, S, \& Dufour, J. (1992). Ascorbic, dehydroascorbic and isoascorbic acid simultaneous determinations by reverse phase ion interaction HPLC. Journal of Food Science, 57(2), 506-511. doi:10.1111/j.1365-2621. 1992.tb05527.x 